\title{
Research of the Optimal Level of Compression by Using Haar Wavelet and Recognition System
}

\author{
Rachid El ayachi ${ }^{1}$, Mohamed Biniz ${ }^{2}$ and Mohamed Fakir ${ }^{3}$ \\ Laboratory of Information Processing and Decision Support (TIAD) \\ Faculty of Sciences and Technics, University Sultan Moulay Slimane. Morocco \\ ${ }^{1}$ rachid.elayachi@usms.ma, ${ }^{2}$ mohamedbiniz@gmail.com,3fakfad@yahoo.fr
}

\begin{abstract}
The Haar wavelet is a lossless compression approach, it is applied in several levels, at each level the size is reduced by a rate of $75 \%$. This reduction produces degradations in the image quality, and consequently the loss of the content of the original image. The purpose of this article is to find the optimal level of compression, which means the level of compression that produces the smallest compressed image that retains the original image content.

The search for the optimal level of compression will be based on a proposed system, this system contains two parts: the first part use the principle of Haar wavelets algorithm to produces the compressed images at several levels and the second part use a recognition system as a criterion control to detect and recognize the content of images.
\end{abstract}

Keyword: Image, Haar wavelets, Compression level, Optimal of compression level, Recognition system

\section{Introduction}

Image treatment is an area which contains several processes like compression. The compression is an algorithm which allows the reduction of size and transmission time of information (image). It is devised into two types: lossless and lossy. Haar wavelet is an example of lossy compressing algorithm which can be applied on several levels. For each level, we have a compressed image. This algorithm was attracted the intention of the scientific community. P. Bhirud and al [1] did a comparative study of various filters of Wavelet Transforms in terms of size and PSNR of images. A. Hazarathaiah and al [2] have presented the construction of new lifting based wavelets by a new method of calculating lifting coefficients. R. El Ayachi and al [3] have proposed a new method based on the Haar wavelet algorithm in order to reduce the computational complexity.

Since Haar wavelets approach produce compressed images in several levels, the optimal level of compression is a level where the resulting compressed image is characterized by: the smallest size and conservation of the original image content.

The research of the optimal level of compression requires tools allowing the control at each level of compression. This control will be realized by a comparison between the original image and the compressed image. In literature, there are several mathematical formulas that can be used, namely: MSE (Mean Square Error), PSNR (peak signal-tonoise ratio) [4] and SSIM (Structural similarity) [5], but these mathematical formulas require the same size for both images.

The problem of the size is resolved by using the segmentation processing. R. El Ayachi and al [6] have adopted the segmentation method as criteria to detect the optimal level of compression in which the number of the regions of the compressed image is still the same

Received (March 6, 2018), Review Result (June 4, 2018), Accepted (June 24, 2018) 
as in the original image. Sadly, this method is not effective in all cases, it has two limitations:

- The first concerns the shape of the segment, because the successive compression generates changes in the shapes of the segments.

- The second focuses on the minimum size of a segment, because successive compressions can produce a segment containing a single pixel, so what is the minimum size of a segment?

To overcome these limitations (shape and size), the solution is to use a recognition system that allows to identify the contents of an image, and consequently the control of the shape and the size of its partitions.

So, the purpose of this paper is to develop a system that allows to produce a compressed image at an optimal level, this system is based on two approaches:

- The first approach, called the Haar wavelets, is used for compression on several levels.

- The second approach, called a recognition system, is adopted to control the content.

The rest of paper is organized as follows: Section 2 gives a description on the use of the Haar wavelet method in image compression. Section 3 is dedicated to the presentation of the operating of a recognition system and its components. Section 4 proposes a system that produces the optimal compression of an input image. Section 5 is devoted to the experimental results obtained. Finally, the conclusion is given in Section 6.

\section{Compression Level using the Haar Wavelet Approach}

Haar wavelet is a compressing algorithm that applies in several levels. At each level, he divides an input image (original image) into four parts (sub-images) [6]; one part contains the compressed image, and the other parts contain detail coefficients for the decompression process. The principle of Haar wavelet algorithm is described in Figure 1.

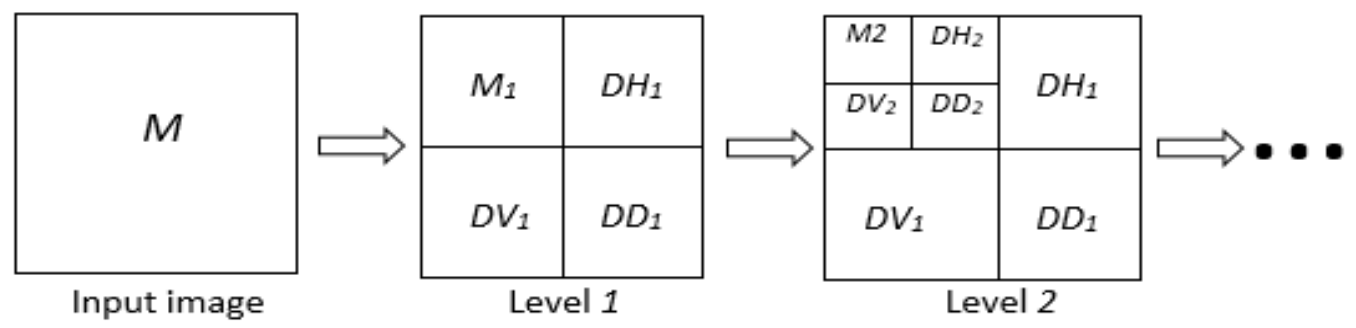

Figure 1. Principle of Haar Wavelets Algorithm

In Figure 1, the description of parameters are:

- $M_{h}$ : Image compressed at level $h$

- $\quad D H_{h}, D V_{h}$ and $D D_{h}$ : Detail coefficients at level $h$

- $h$ : Compressing level

For each level, the Haar wavelet algorithm uses the following formula:

$$
S c_{h}=t\left(A_{h}\right) * M_{h-1} * A_{h}
$$


where

- $h$ : Compression level

- $S c_{h}$ : Compressed image at level $h$

- $A_{h}$ : Haar wavelet coefficients at level $h$

- $t\left(A_{h}\right)$ : Transposed of $A_{h}$

- $M_{h-1}$ : Original image for level $h$

The Haar wavelet coefficients of $A$ can be computed using the formulas listed below:

$$
\left\{\begin{array}{l}
A_{i j}=1 / 2 \text { if } j \leq \mathrm{N} / 2 \text { and } \mathrm{i}=2 * \mathrm{j}+1 \text { or } \mathrm{i}=2 * \mathrm{j} \\
A_{i j}=1 / 2 \text { if } \mathrm{N} / 2<j \leq \mathrm{N} \text { and } \mathrm{i}=2 *(\mathrm{j}-\mathrm{N} / 2)-1 \\
A_{i j}=-1 / 2 \text { if } \mathrm{N} / 2<j \leq \mathrm{N} \text { and } \mathrm{i}=2 *(\mathrm{j}-\mathrm{N} / 2) \\
A_{i j}=1 \text { if } \mathrm{i}>N \\
A_{i j}=0 \text { else } \\
\text { where }
\end{array}\right.
$$

- $\quad N$ : Number of columns

- $\quad i, j$ : Positive integers

\section{Recognition System}

Pattern recognition is an area that focuses on identifying the information content (Image for this paper) [7]. This content can have several forms: text or graphic patterns. It has several applications: disease detection [8], biometric recognition [9], signature recognition [10], barcode recognition [11], etc.

Recognition system is a system that interest to detect and recognize the content of input information.

Generally, it contains four components (Figure 2):

- The first component is named Acquisition; it is used to acquire an image at the input of the system.

- The second component is named Pretreatment; it contains a set of functions applied on the input image to produce a clean version that well be available for the next steps.

- The third component is named Extraction; it allows to extract a set of features that characterize the image content.

- The fourth component is named Classification; it focuses on the identification of the image content using the features extracted in the previous step.

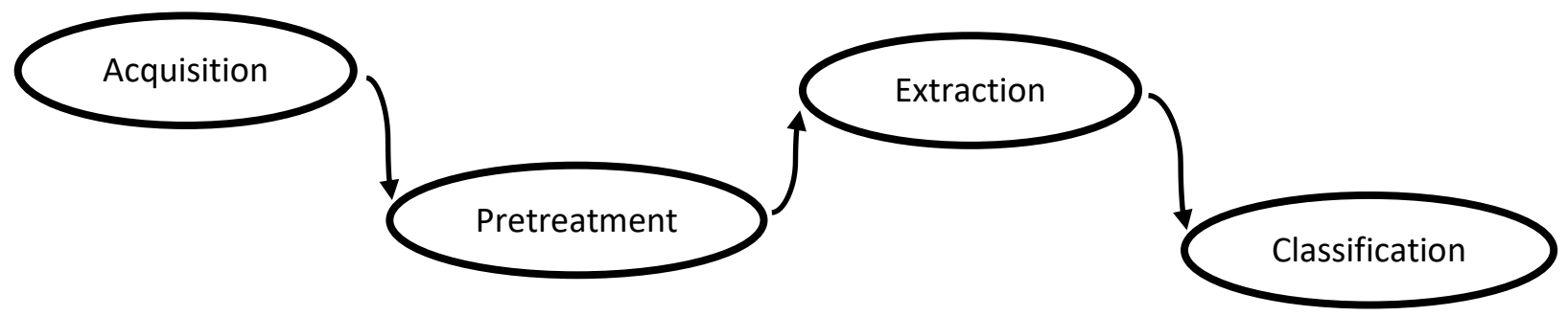

Figure 2. Recognition System 


\section{Proposed System}

The proposed system allows to produce the optimal level of compression for an original image, it contains two modules (Figure 3): Compression and Control. The first is used to generate a compressed image at several levels using Haar wavelets. The second is adopted to control and compare the content of both images (compressed image and original image) by using the recognition system.

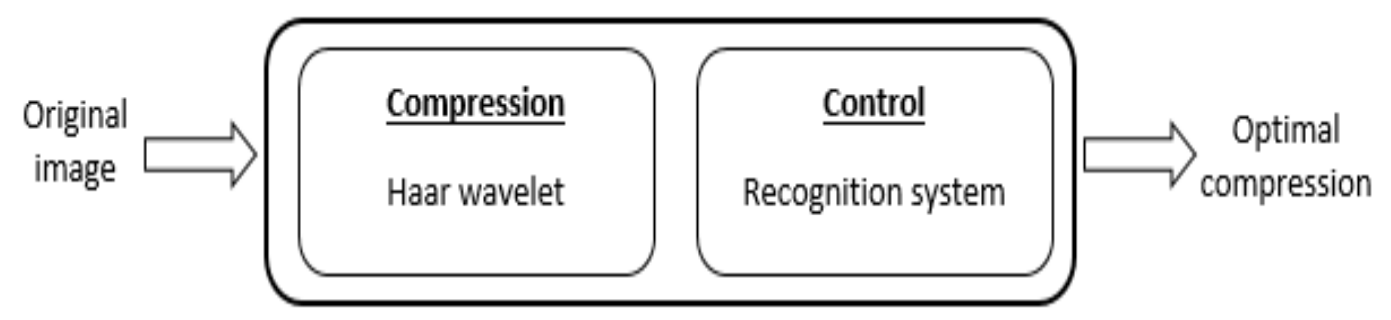

Figure 3. Proposed System

This system has several advantages, namely:

- The obtaining of a compressed image at an optimal level; the production of the smallest image conserving the contents of the original image, this allows a reduction of the storage size, the transmission time and the time for a postprocessing.

- The comparison of two images of different sizes; in the literature there exist only the methods allowing a comparison between two images of the same sizes.

\subsection{Operating of the Proposed System}

The operating of the proposed system follows a set of steps described as follows:

- Step 1: Acquiring an original image to compress.

- Step 2: Applying the recognition system to the original image to build the reference database that will be used in the control process.

- Step 3: Computing of the compressed image at level $h$ by using Haar wavelets $(h=1,2, \ldots)$.

- Step 4: Applying the recognition system to the compressed image (obtained in step 3) to identify the content.

- Step 5: Similarity decision of the system

- Go to Step 3 in case the two contents are similar.

- Stopping the system in the opposite case, this gives that the optimal level of compression is the predecessor level.

The steps described above are schematized in the following organization chart: 


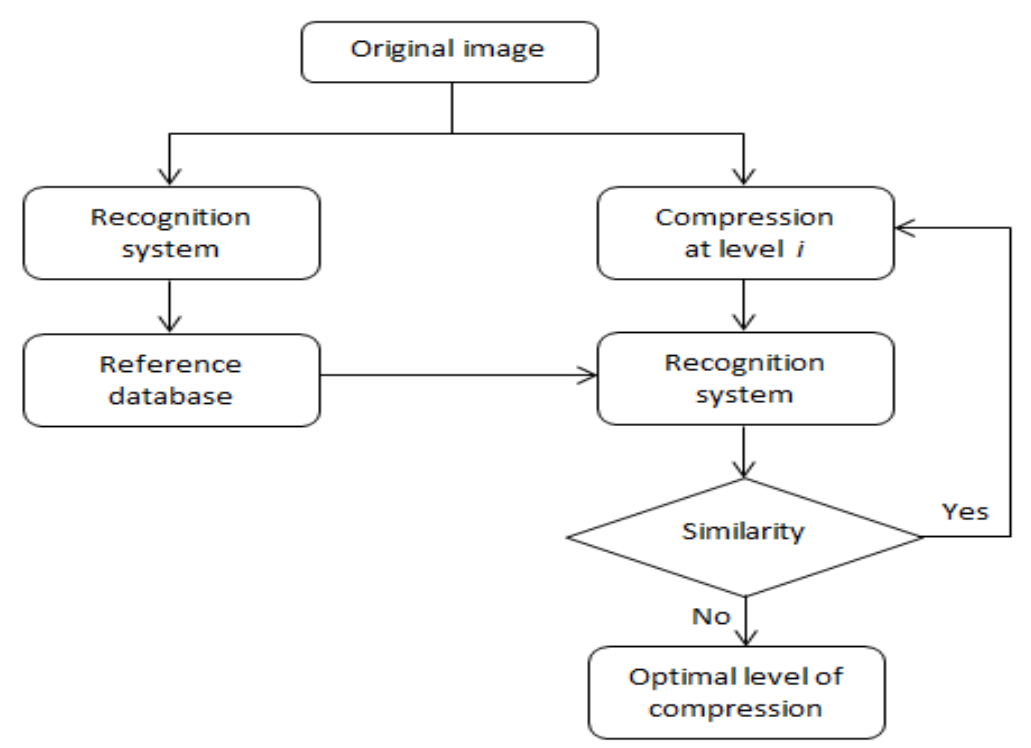

Figure 4. Organization Chart of Proposed System

\subsection{Components of the Recognition System}

The recognition system (Figure 5) used in this paper contains the following processes:

- Pretreatment:

- Binarization: it is a process that converts the picture pixels into two colors (white and black), this treatment is used for text image.

- Normalization: it is a process that eliminate unnecessary areas in a picture by using histogram approach [12].

- Segmentation: it is a process that separates the picture into homogeneous areas by using the method of segmentation by thresholding [13].

- Extraction: it is a process that extract features from segmented areas, these features must be invariant to rotation, translation and scale. In this treatment, invariant moments (Hu moments) are used [14].

- Classification: it is a process that recognizes the content of segmented areas basing on reference database. KNN method (K-Nearest Neighbors) is adopted in this step [14].

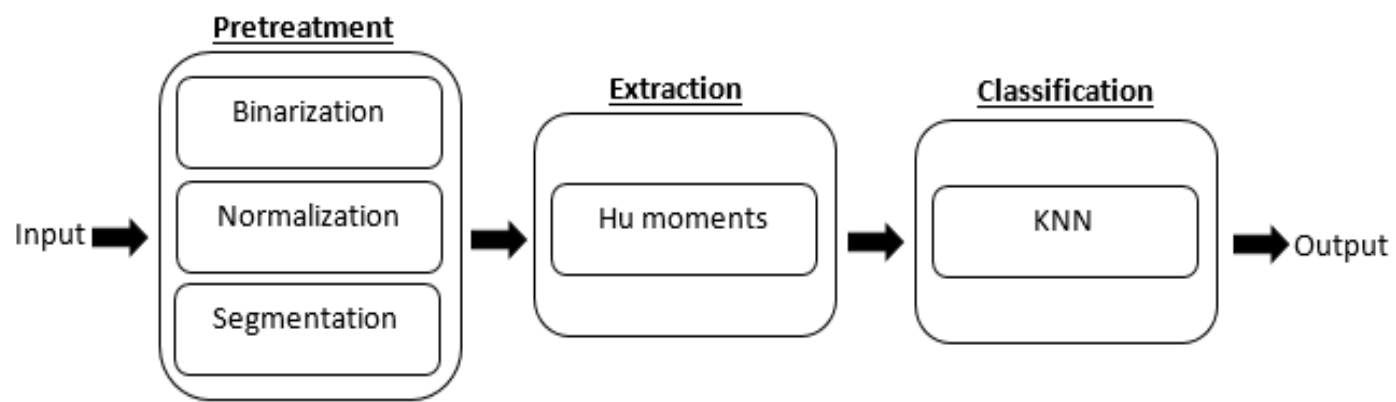

Figure 5. Components of the Recognition System 


\section{Results}

Haar wavelets is a compression algorithm applied at several levels. Figure 6 shows the results for an example of use up to level 8 .

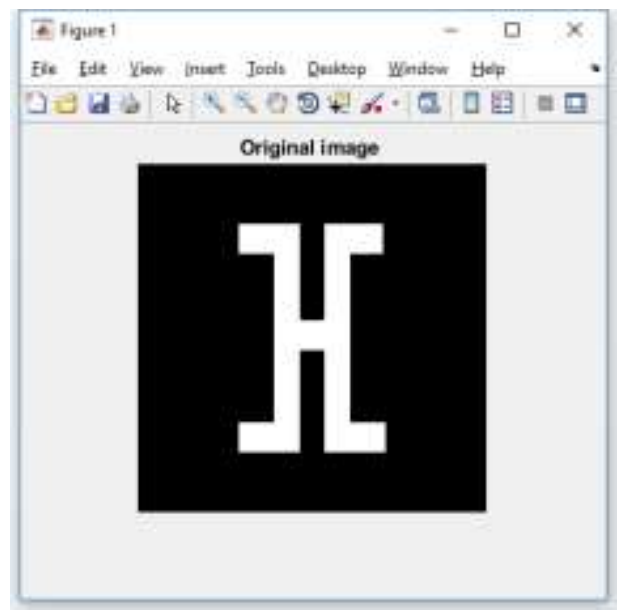

Original Image

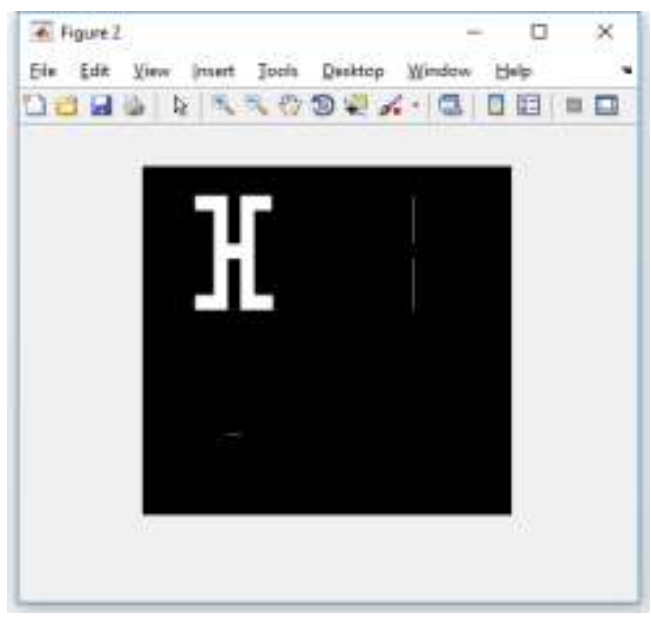

Level 1

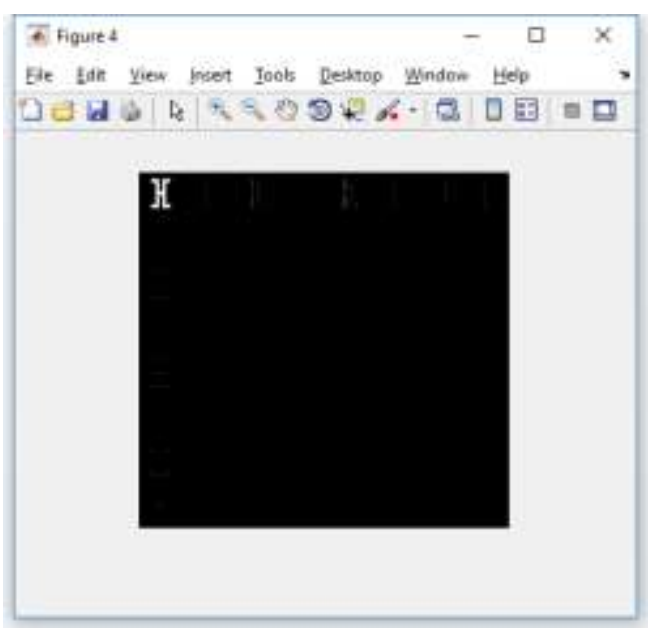

Level 3

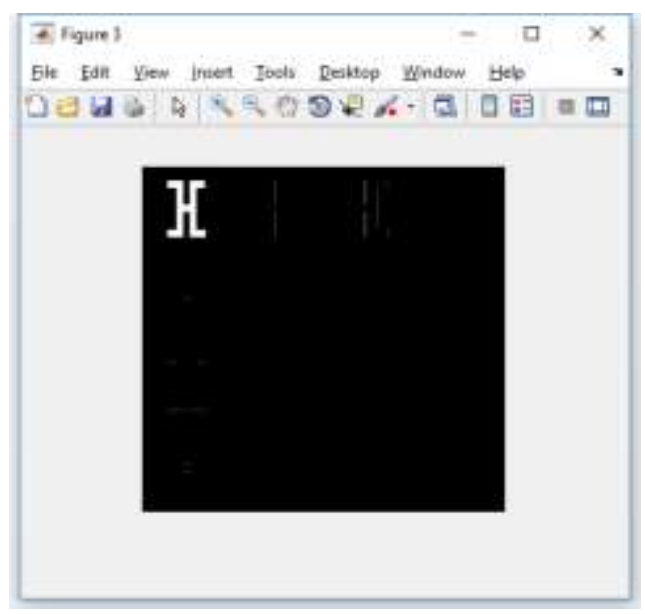

Level 2

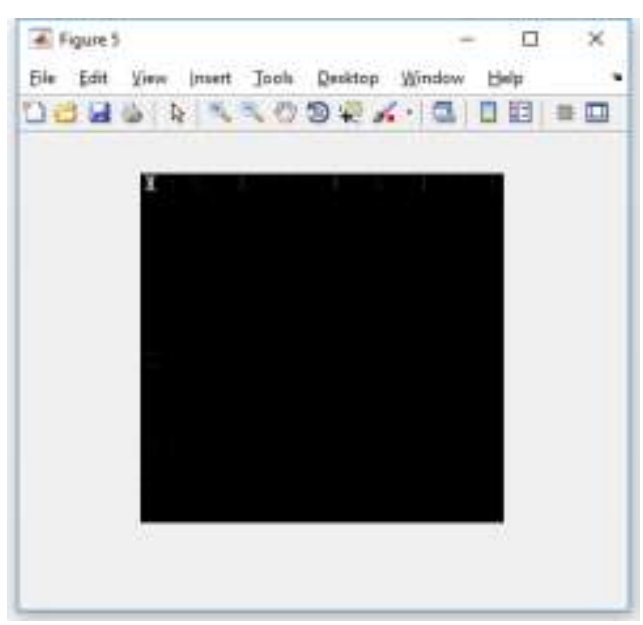

Level 4 


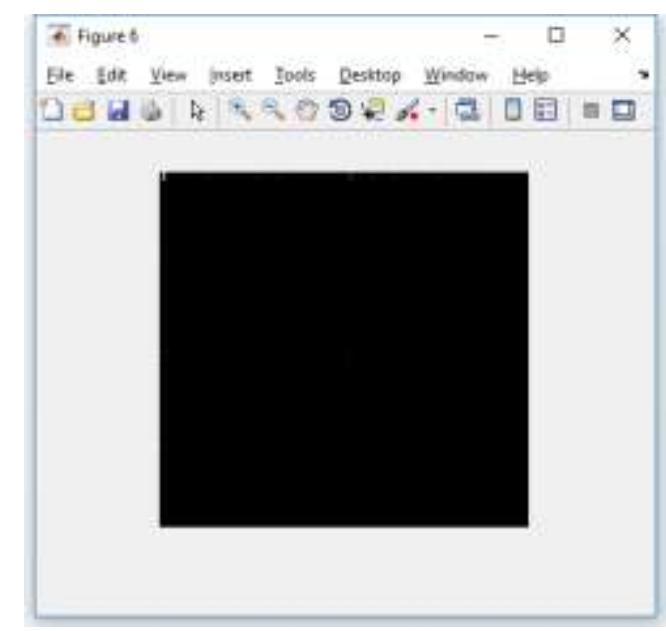

Level 5

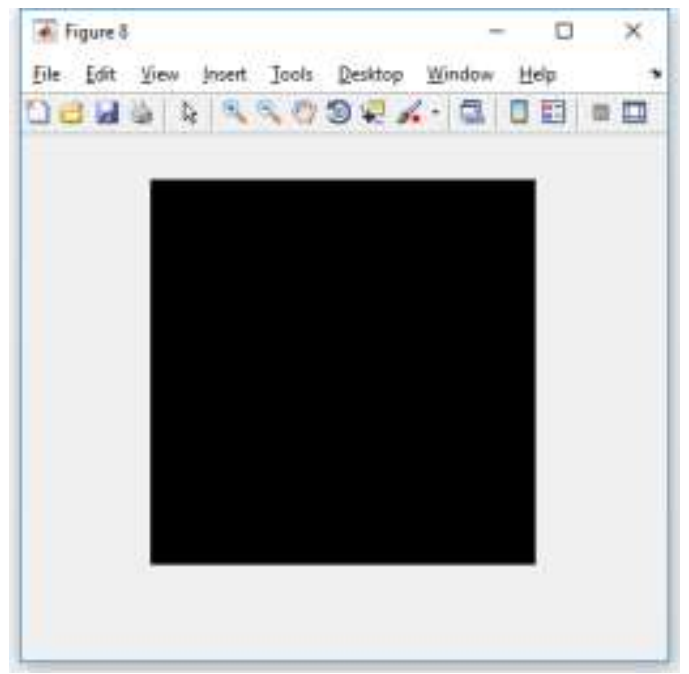

Level 7

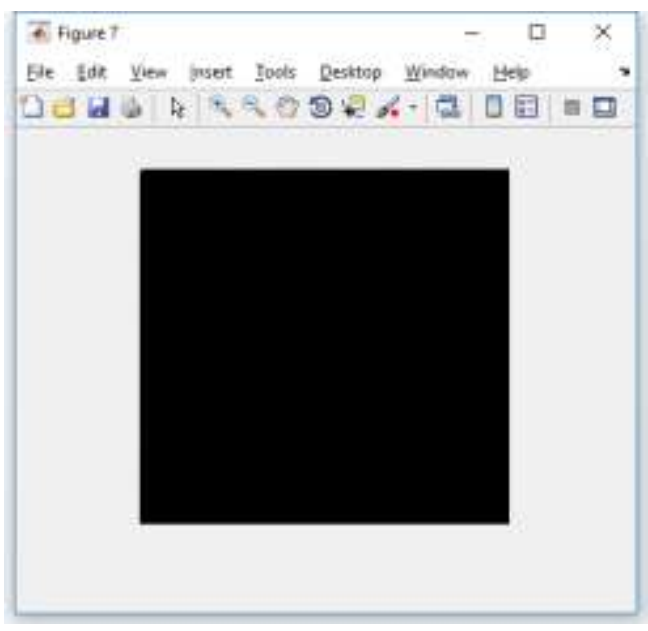

Level 6

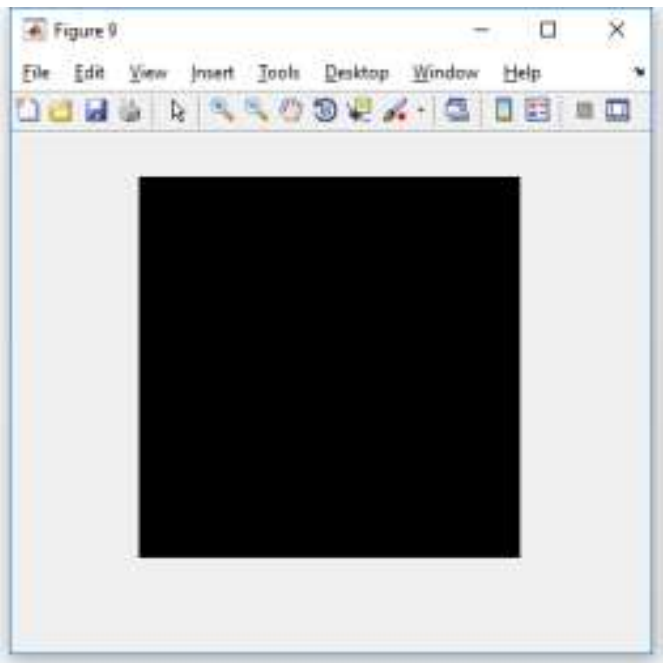

Level 8

Figure 6. Application of Haar Wavelets for 7 Levels

From Figure 6, the following remarks can be made:

- From level 6, the content of the original image is absent due to the small size of the compressed image (a few pixels).

- From level 6, the compression levels are useless because the original content is lost.

- Level 5 contains an ambiguous information segmentation as a control criterion is not enough and it is useless to continue compression to the next levels.

The results presented above show the importance of adding a recognition system to control the content, hence obtaining the optimal level of compression. Figure 7 shows the results obtaining by using the proposed system composed of two modules: Compression and Recognition system. 


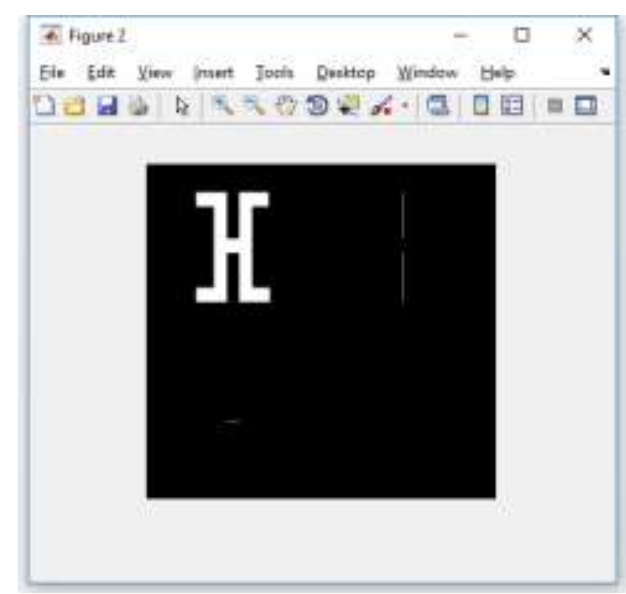

Level 1

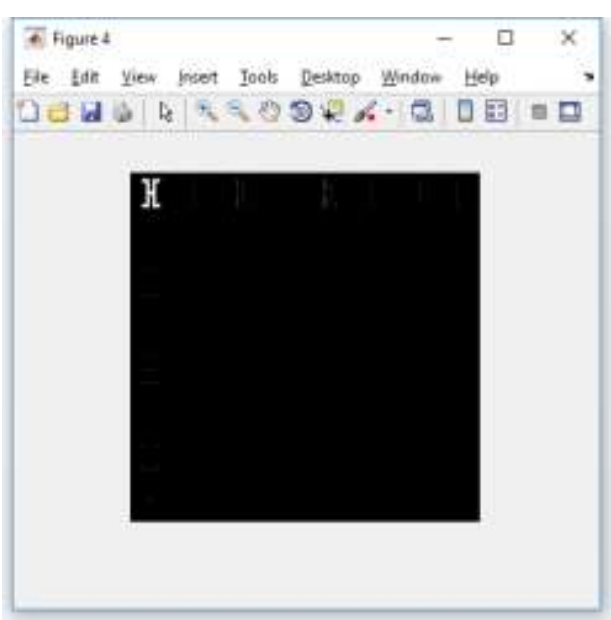

Level 3

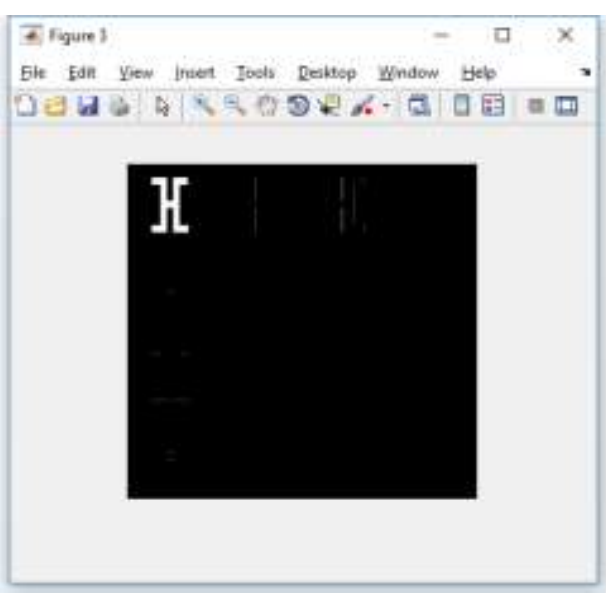

Level 2

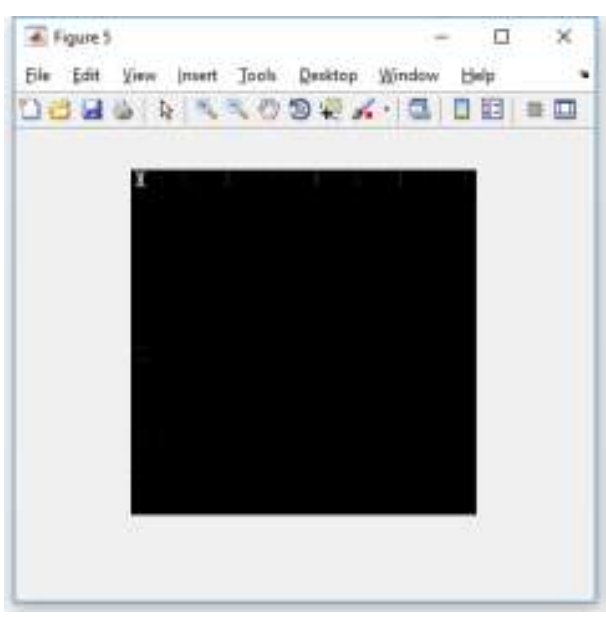

Level 4

Figure 7. Application of Proposed System

The figure above shows the multi-level compressed images that are similar to the contents of the original image.

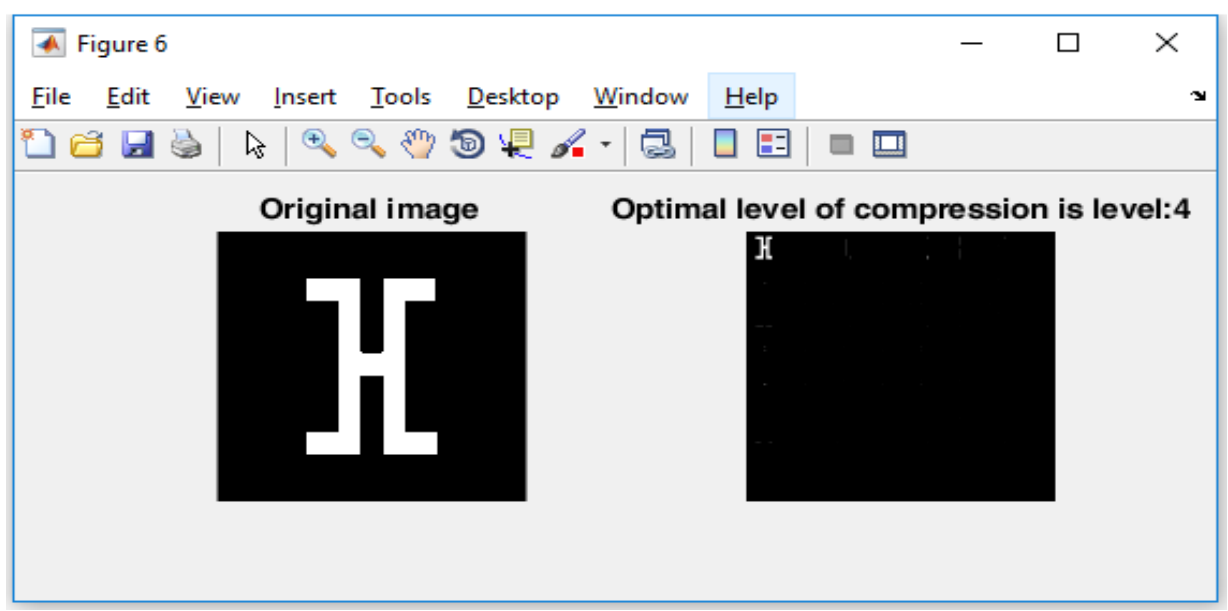

Figure 8. Result of the Optimal Level of Compression using Proposed System 
Figure 8 shows an example of the application of the proposed system; the displayed result means (for the input image) that the optimal level of compression is level 4, this level corresponds to the smallest image retaining the content of the original image.

The proposed system is tested on the database [15] that contains 2175 Tifinagh printed characters with different sizes and styles.

\section{Conclusion}

Compression is a process that reduces the size of an image. The idea discussed in this paper is the search for the smallest image that retains the content of an original image. This idea is solved using a proposed system.

In this work, a proposed system is presented, it allows to compute the optimal level of compression. This system is composed of two modules: Compression and Recognition system. The first module is used to calculate the compressed images at several levels using the principle of Haar wavelets algorithm. But the second module is used as a control criterion to survey the content of images.

The results obtained justify the effectiveness of the system developed and the importance of using a recognition system as a tool for controlling the content of images.

\section{References}

[1] P. Bhirud and N. Prabhu, "Performance Evaluation of Filters of Discrete Wavelet Transforms for Biometrics", International Journal of Informatics and Communication Technology (IJ-ICT), vol. 3, no. 2, (2014) June, pp. 97-102.

[2] A. Hazarathaiah and B. Prabhakara Rao, "Medical Image Compression using Lifting based New Wavelet Transforms", International Journal of Electrical and Computer Engineering (IJECE), vol. 4, no. 5, (2014) October, pp. 741-750.

[3] R. El Ayachi, B. Bouikhalene and M. Fakir, "New Image Compression Algorithm using Haar Wavelet Transform", International Journal of Informatics and Communication Technology (IJ-ICT), vol. 6, no. 1, (2017) April, pp. 43-48.

[4] A. Tamtaoui and D. Aboutajdine, "Visual quality measure of the compressed images", Traitement du Signal, vol. 20, no. 1, (2003).

[5] A. Bhattacharya and T. Chatterjee, "An Estimation Method of Measuring Image Quality for Compressed Images of Human Face", International Journal of Computer Trends and Technology (IJCTT), vol. 7, no. 3, (2014) January.

[6] R. El Ayachi, M. Gouskir and M. Baslam, “Application of Haar Wavelets on Medical Images”, Journal of Electronic Commerce in Organizations, vol. 13, no. 2, (2015) April-June, pp. 41-49.

[7] K. Vora, A. Shah and J. Mehta, "A Review Paper on Currency Recognition System", International Journal of Computer Applications, (0975-8887), vol. 115, no. 20, (2015) April.

[8] R. Kaur and M. Kaur, "A Brief Review on Plant Disease Detection using in Image Processing", International Journal of Computer Science and Mobile Computing, vol. 6, no. 2, (2017) February, pp. 101-106.

[9] L. Bouhou, R. El Ayachi, M. Baslam and M. Oukessou, "Face Detection in a Mixed-Subject Document", International Journal of Electrical and Computer Engineering (IJECE), vol. 6, no. 6, (2016) December, pp. 2828-2835.

[10] V. Malik and A. Arora, "A Review Paper on Signature Recognition”, International Journal for Research in Applied Science \& Engineering Technology (IJRASET), vol. 3, no. VI, (2015) June.

[11] N. M. Z. Hashim, N. A. Ibrahim, N. M. Saad, F. Sakaguchi and Z. Zakaria, "Barcode Recognition System”, International Journal of Emerging Trends \& Technology in Computer Science (IJETTCS), vol. 2, no. 4, (2013) July-August.

[12] R. El Ayachi, M. Fakir and B. Bouikhalene, "Recognition of TIFINAGHE Characters Using A multilayer Neural Network", International Journal Of Image Processing (IJIP), vol. 5, no. 2, (2011), pp. 109-118.

[13] N. Senthilkumaran and S. Vaithegi, "Image Segmentation by using Thresholding Techniques for Medical Images", Computer Science \& Engineering: An International Journal (CSEIJ), vol. 6, no. 1, (2016) February.

[14] M. Oujaoura, R. El Ayachi, B. Minaoui, M. Fakir, B. Boukhalene and O. Bencharef, "Invariant Descriptors and Classifiers Combination for Recognition of Isolated Printed Tifinagh Characters", IJACSA Special Issue on Selected Papers from Third international symposium on Automatic Amazigh processing (SITACAM' 13), (2013), pp. 22-28. 
[15] Y. Ait Ouguengay and M. Taalabi, “Elaboration d'un réseau de neurons artificiels pour la reconnaissance optique de la graphie amazighe: Phase d'apprentissage", Systèmes intelligents-Théories et applications, Paris: Europia, cop. 2009 (impr. au Maroc), ISBN-102909-285553, (2009).

\section{Authors}

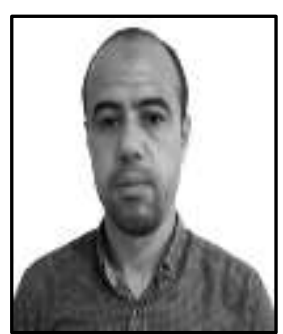

Rachid El Ayachi, obtained a degree in Master of Informatic Telecom and Multimedia (ITM) in 2006 from the Faculty of Sciences, Mohammed V University (Morocco) and a Ph.D. degree in computer science from the Faculty of Science and Technology, Sultan Moulay Slimane University (Morocco). He is currently a member of laboratory TIAD and a professor at the Faculty of Science and Technology, Sultan Moulay Slimane University, Morocco. His research focuses on image processing, pattern recognition, machine learning, semantic web, ...etc.

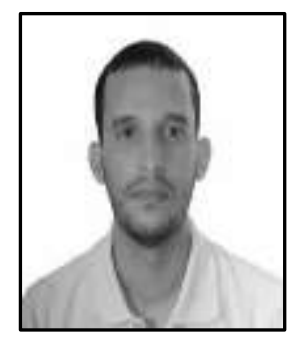

Mohamed Biniz, received his master's degree in business intelligence in 2014 from the Faculty of Science and Technology, University Sultan Moulay Sliman Beni-Mellal. He is currently a $\mathrm{PhD}$ degree sutdent. His research activities are located in the area of the semantic web engineering specifically, it deals with the research question of the evolution of ontology, big data, natural language processing, dynamic programming, etc.

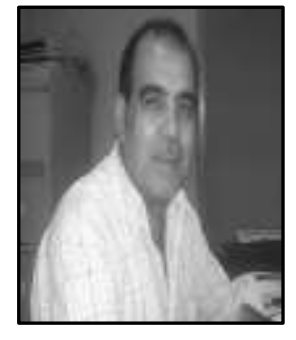

Mohamed Fakir, obtained a degree in Master of Electrical Engineering from Nagaoka University of Technology in 1991 and a $\mathrm{PhD}$ degree in electrical engineering from the University of Cadi Ayyad, Morocco. He was a team member in Hitachi Ltd., Japan between 1991 and 1994. He is currently a professor at the Faculty of Science and Technology, University Sultan Moulay Slimane, Morocco. His research interest includes image processing, pattern recognition, semantic web, big data and artificial intelligence. 